\title{
Expressional analysis of immune-related miRNAs in breast milk
}

\author{
R.S. Na ${ }^{1,2}$, G.X. E ${ }^{2}$, W. Sun ${ }^{2}$, X.W. Sun ${ }^{2}$, X.Y. Qiu ${ }^{2}$, L.P. Chen ${ }^{2}$ and \\ Y.F. Huang ${ }^{2 *}$ \\ ${ }^{1}$ Chongqing Academy of Animal Sciences, Chongqing, China \\ ${ }^{2}$ College of Animal Science and Technology, Southwest University, \\ Chongqing Key Laboratory of Forage and Herbivore, Chongqing Engineering \\ Research Center for Herbivores Resource Protection and Utilization, \\ Chongqing, China \\ *Corresponding author: Y.F. Huang \\ E-mail: h67738337@swu.edu.cn
}

Genet. Mol. Res. 14 (3): 11371-11376 (2015)

Received January 3, 2015

Accepted May 15, 2015

Published September 25, 2015

DOI http://dx.doi.org/10.4238/2015.September.25.4

\begin{abstract}
Immune-related miRNAs in breast milk are extracellular miRNAs that are related to immune organ development and regulation of the immune function in infants and young animals. The goal of this study was to compare the expression levels of five immune-related miRNAs in breast milk in black goats, humans, and dairy cattle. The miRNAs from milk were extracted and the expression levels were assessed using quantitive RT-PCR methods. MiR-146, miR-155, miR-181a, miR-223, and miR-150 were all detected in Dazu black goat milk, and these miRNAs were significantly more highly expressed in colostrum than in mature milk of goats $(\mathrm{P}<0.01)$, except for miR-150. Further, all five miRNAs were expressed in human colostrum, but patterns differed from those in goats: miR-146 and miR-155 were highly expressed $(\mathrm{P}<0.01)$ in human colostrum, whereas miR-223 was abundant in goat colostrum $(\mathrm{P}<0.01)$. In addition, five miRNAs were significantly higher in bovine mature milk than in goat milk $(\mathrm{P}<0.01)$. Taken together,
\end{abstract}


these results confirm that immune-related miRNAs are rich in breast milk with different expression levels depending on the lactation phase and species.

Key words: MicroRNA; Colostrum; Mature milk; Goat

\section{INTRODUCTION}

MicroRNA (miRNA) is an endogenous small noncoding RNA, a newly identified class of post-transcriptional regulatory molecules involved in cell proliferation, differentiation, apoptosis, signal transduction, organ development, and other important life processes (Chen et al., 2004; Pencheva et al., 2012). miRNAs are present in various body fluids and selectively packaged inside exosomes, which are actively delivered into recipient cells and regulate target gene expression and recipient cell function, but the mechanisms are not very clear (Weber et al., 2010; Lasser et al., 2012). The unique expression profile of body fluid-specific miRNAs can serve as novel indicators or biomarkers and also make possible new standards for the quality control of commercial products (Chen et al., 2010).

In recent years, miRNAs have been found in breast milk of humans and livestock (Kosaka et al., 2010; Gu et al., 2012a,b; Izumi et al., 2012; Jabed et al., 2012; Li et al., 2012b; Zhou et al., 2012; Bai et al., 2013). Human milk is rich in immune-related miRNAs, including miR-181a, miR-181b, miR-155, miR-17, miR-92a, miR-125b, miR-146a, miR146b, miR-223, let-7i, and miR-150 (Kosaka et al., 2010; Weber et al., 2010; Zhou et al., 2012) Among these, miR-181a is an intrinsic modulator of T-cell sensitivity and selection (Li et al., 2007, 2012a); miR-146 controls toll-like receptor and cytokine signaling and promotes cluster of designation cell differentiation (Taganov et al., 2006); miR-150 controls B cell differentiation and is important for pre- and pro-B cell formation or function (Xiao et al., 2007; Zhou et al., 2007). Immune-related miRNAs, including miR-155 and miR-223, are also enriched in porcine and bovine breast milk (Gu et al., 2012a; Izumi et al., 2012). miR-155 regulates T-helper cell differentiation and the germinal center reaction to produce an optimal T-cell-dependent antibody response (Rodriguez et al., 2007; Thai et al., 2007); miR-223 acts to fine-tune granulocyte production and the inflammatory response (Johnnidis et al., 2008; Chen et al., 2012).

In this study, we analyzed expression levels of immune-related miRNAs in milk of black goats, cattle, and humans. We expect this research to stimulate further studies of miRNAs expression profiles in animal milk.

\section{MATERIAL AND METHODS}

The highly prolific Dazu black goats used in this experiment were obtained from the College of Animal Science and Technology, Southwest University, Chongqing, China. They were listed as one of the 156 nationally protected domestic animals by the Chinese government in 2014. Goat milk samples were collected at two lactating stages ( 7 and 30 days) from three ewes after normal delivery. Bovine milk was taken from three dairy cows at 3 months postpartum. Human breast milk samples were collected from three volunteers with infants aged 7 days or younger. All samples were immediately frozen in liquid nitrogen and stored at $-80^{\circ} \mathrm{C}$ until analysis. Small RNAs were isolated using RNAiso for Small RNA (Takara, 
D340), and reverse transcribed by One Step PrimeScript miRNA cDNA Synthesis kit (Takara, D350).

Real-time PCR was performed using a standard SYBR Green PCR kit (Bio-Rad) on a BIO-RAD CFX96 Touch ${ }^{\mathrm{TM}}$ Real-Time PCR Detection System. The $10 \mu \mathrm{L}-\mathrm{PCR}$ contained $5 \mu \mathrm{L}$ SsoAdvance SYBR Green Supermix (Bio-Rad), $0.5 \mu \mathrm{L}$ cDNA, $0.4 \mu \mathrm{M}$ miRNA specific forward primer (see Table 1), and $0.4 \mu \mathrm{M}$ universal reverse primer. The samples were incubated on a 96-well plate at $95^{\circ} \mathrm{C}$ for $30 \mathrm{~s}$, followed by 40 cycles of $95^{\circ} \mathrm{C}$ for $5 \mathrm{~s}$ and the optimized annealing temperature for $30 \mathrm{~s}$.

\begin{tabular}{|c|c|c|}
\hline miRNA & Forward primer & Reverse primer \\
\hline miR-146 & 5'-gctgagaactgaattccataggttg-3' & D352 (Takara) \\
\hline miR-150 & $5^{\prime}$-tctcccaaccettgtaccagtgt-3' & D352 (Takara) \\
\hline miR-155 & $5^{\prime}$-gcttaatgctaatcgtgataggggt3' & D352 (Takara) \\
\hline miR-181a & $5^{\prime}$-aacattcaacgctgtcggtga- $3^{\prime}$ & D352 (Takara) \\
\hline miR-223 & 5'-gctgtcagttgcaaatacccca-3' & D352 (Takara) \\
\hline
\end{tabular}

\section{RESULTS}

We found miR-146, miR-150, miR-155, miR-181a, and miR-223 expressed in Dazu black goat milk samples collected during different lactation periods. Among these, miR-146, miR-155, miR-181a, and miR-223 were strongly expressed in goat colostrum compared with that in mature milk $(\mathrm{P}<0.01)$, whereas the lymphocyte-regulated miR-150, which was not abundant in goat colostrum, was abundantly expressed in mature breast milk (see Figure 1).

We also found differences in immune-related miRNA expression in goat, human, and dairy cattle milk. miR-146, miR-155, and miR-181a were rich in human colostrum compared with that in goats; in particular, miR-146 and miR-155 were extremely highly expressed in human colostrum $(\mathrm{P}<0.01)$. The expression of lymphocyte-regulated miR-150 was not different in goat and human colostrum. Only miR-223 was much more highly expressed in goat colostrum than in human milk $(\mathrm{P}<0.01)$ (see Figure 2), whereas the five immune-related miRNAs were all highly expressed in the mature milk of mature cattle when compared with the mature milk of mature black goats $(\mathrm{P}<0.01)$ (see Figure 3$)$.

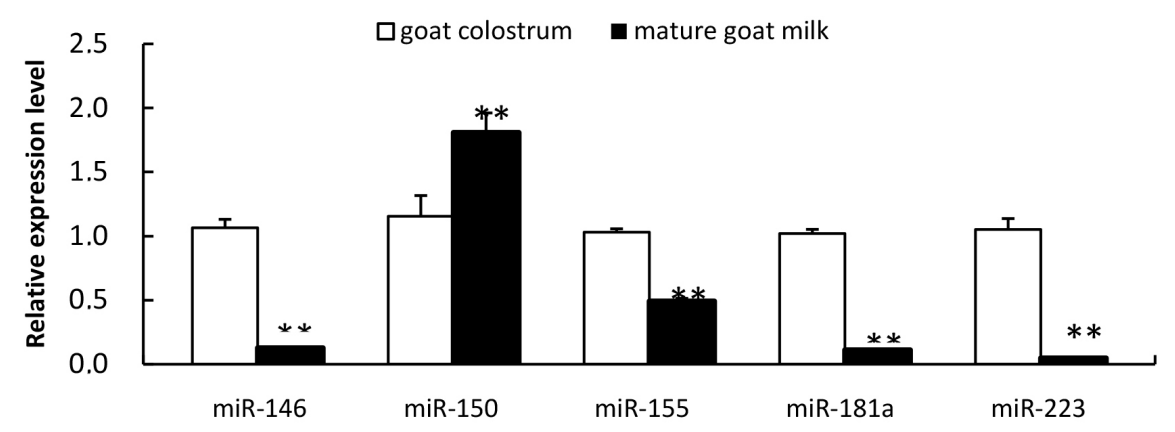

Figure 1. Comparison of the expression levels of the five immune-related miRNAs in goat colostrum and mature milk. The y axes show arbitrary units representing relative miRNA expression levels. The expression levels of miRNAs in colostrum were arbitrarily set to 1.0 . Results are reported as means \pm SEM of three independent experiments $(* * \mathrm{P}<0.01)$. 


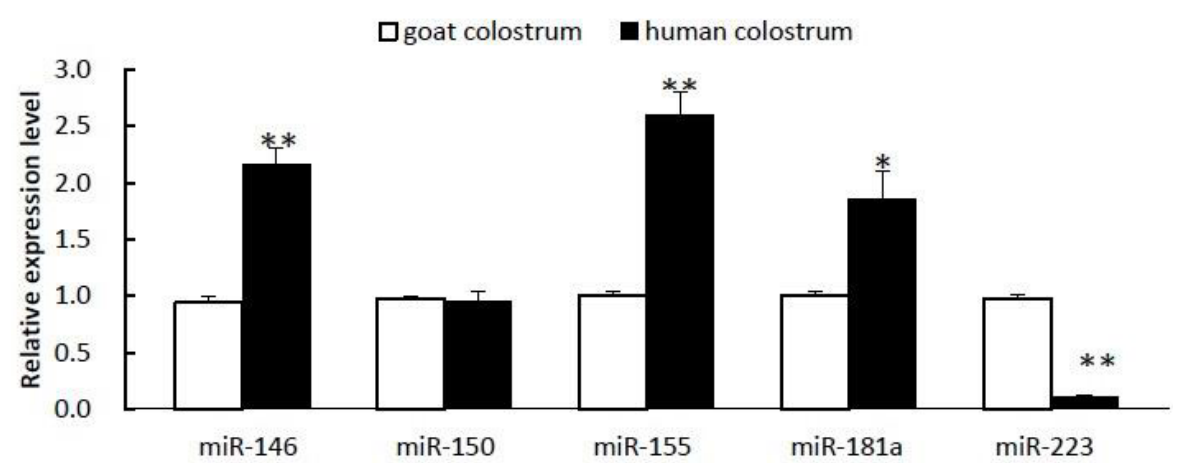

Figure 2. Expressional analysis of five immune-related miRNAs in goat and human colostrum. The y-axis shows arbitrary units representing relative miRNA expression levels. The expression levels of miRNAs in Dazu black goat colostrum were arbitrarily set to 1.0. Results are reported as means $\pm \mathrm{SEM}$ of three independent experiments $(* \mathrm{P}<0.05, * * \mathrm{P}<0.01)$.

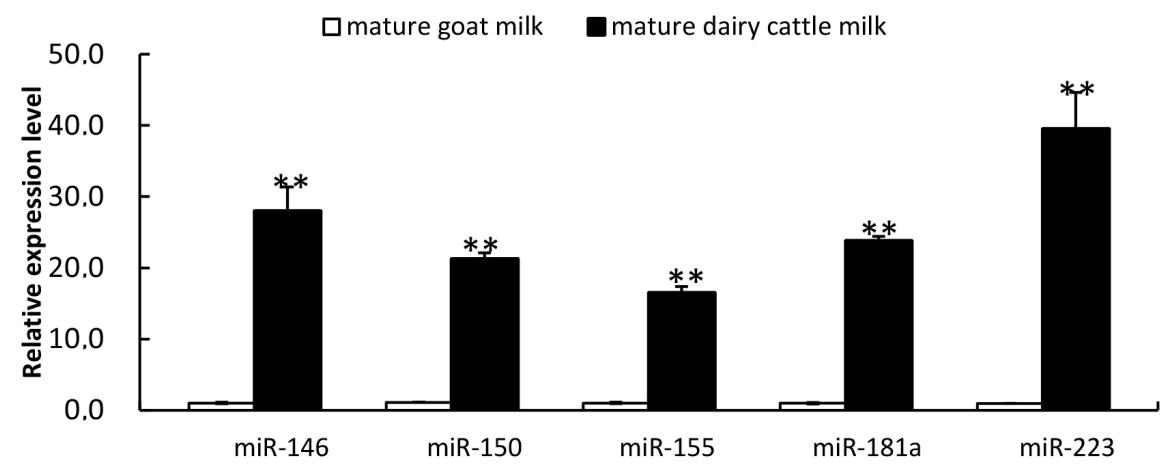

Figure 3. Comparison of the expression levels of the five immune-related miRNAs in mature goat and dairy cattle milk. The y-axis shows arbitrary units representing relative miRNA expressional levels. The expression levels of miRNAs in mature Dazu black goat milk were arbitrarily set to 1.0. Results are reported as means \pm SEM of three independent experiments $(* * \mathrm{P}<0.01)$.

\section{DISCUSSION}

Some of the functions of immune-related miRNAs are clarified in the present report; however, the functions of many of these in breast milk are still unknown. In our study, we detected five immune-regulatory miRNAs in Dazu black goat milk and confirmed their presence. Further, miR-155, miR-181a, miR-146, and miR-223 were significantly more highly expressed in colostrum than in mature milk in goat. Our results are similar to those from another study of miRNAs in bovine milk (Izumi et al., 2012). Interestingly, the expression level of miR-150 increased in mature milk, which contrasts with expression of other immune-related miRNAs. As we know, miR-150, expressed in mature B and T cells, controls B-cell differentiation by targeting the transcription factor c-Myb and blocks early B-cell development when expressed prematurely (Xiao et al., 2007; Zhou et al., 2007). These findings indicate goat milk may modulate the development of the immune system in lambs.

Another aim of our study was to compare the expression distribution of immune- 
related miRNAs in goat, cow, and human milk. The expression level of miR-155, miR-146, and miR-181a in human colostrum was found to be higher than that in goat colostrum, which supports the earlier finding that immune-related miRNAs are abundant in human breast milk (Kosaka et al., 2010). Five selected miRNAs were all extremely highly expressed in mature cow milk compared to that in black goat mature milk. We think the expression differences in breast milk among black goat, human, and cow milk will provide data for studying the function of their immune-related miRNAs and will help in developing formula for infant goats.

\section{ACKNOWLEDGMENTS}

Research supported by the Chongqing Fundamental Research Funds (\#2013cstc-jbby-00909) and the Chongqing Postdoctoral Sustentation Fund (\#Xm201335).

\section{REFERENCES}

Bai WL, Yin RH, Yang RJ, Khan WA, et al. (2013). Technical note: identification of suitable normalizers for microRNA expression analysis in milk somatic cells of the yak (Bos grunniens). J. Dairy Sci. 96: 4529-4534.

Chen CZ, Li L, Lodish HF and Bartel DP (2004). MicroRNAs modulate hematopoietic lineage differentiation. Science 303: 83-86.

Chen Q, Wang H, Liu Y, Song Y, et al. (2012). Inducible microRNA-223 down-regulation promotes TLR-triggered IL-6 and IL-1beta production in macrophages by targeting STAT3. PLoS One 7: e42971.

Chen X, Gao C, Li H, Huang L, et al. (2010). Identification and characterization of microRNAs in raw milk during different periods of lactation, commercial fluid, and powdered milk products. Cell Res. 20: 1128-1137.

Gu Y, Li M, Wang T, Liang Y, et al. (2012a). Lactation-related microRNA expression profiles of porcine breast milk exosomes. PLoS One 7: e43691.

Gu YR, Liang Y, Gong JJ, Zeng K, et al. (2012b). Suitable internal control microRNA genes for measuring miRNA abundance in pig milk during different lactation periods. Genet. Mol. Res. 11: 2506-2512.

Izumi H, Kosaka N, Shimizu T, Sekine K, et al. (2012). Bovine milk contains microRNA and messenger RNA that are stable under degradative conditions. J. Dairy Sci. 95: 4831-4841.

Jabed A, Wagner S, McCracken J, Wells DN, et al. (2012). Targeted microRNA expression in dairy cattle directs production of beta-lactoglobulin-free, high-casein milk. Proc. Natl. Acad. Sci. U. S. A. 109: 16811-16816.

Johnnidis JB, Harris MH, Wheeler RT, Stehling-Sun S, et al. (2008). Regulation of progenitor cell proliferation and granulocyte function by microRNA-223. Nature 451: 1125-1129.

Kosaka N, Izumi H, Sekine K, and Ochiya T (2010). microRNA as a new immune-regulatory agent in breast milk. Silence 1: 7.

Lasser C, Eldh M and Lotvall J (2012). Isolation and characterization of RNA-containing exosomes. J. Vis. Exp. e3037.

Li H, Hui L and Xu W (2012a). miR-181a sensitizes a multidrug-resistant leukemia cell line K562/A02 to daunorubicin by targeting BCL-2. Acta Biochim. Biophys. Sin. 44: 269-277.

Li QJ, Chau J, Ebert PJ, Sylvester G, et al. (2007). miR-181a is an intrinsic modulator of T cell sensitivity and selection. Cell 129: 147-161.

Li Z, Lan X, Guo W, Sun J, et al. (2012b). Comparative transcriptome profiling of dairy goat microRNAs from dry period and peak lactation mammary gland tissues. PLoS One 7: e52388.

Pencheva N, Tran H, Buss C, Huh D, et al. (2012). Convergent multi-miRNA targeting of ApoE drives LRP1/LRP8dependent melanoma metastasis and angiogenesis. Cell 151: 1068-1082.

Rodriguez A, Vigorito E, Clare S, Warren MV, et al. (2007). Requirement of bic/microRNA-155 for normal immune function. Science 316: 608-611.

Taganov KD, Boldin MP, Chang KJ and Baltimore D (2006). NF-kappaB-dependent induction of microRNA miR-146, an inhibitor targeted to signaling proteins of innate immune responses. Proc. Nat. Acad. Sci. USA 103: 12481-21486.

Thai TH, Calado DP, Casola S, Ansel KM, et al. (2007). Regulation of the germinal center response by microRNA-155. Science 316: 604-608.

Weber JA, Baxter DH, Zhang S, Huang DY, et al. (2010). The microRNA spectrum in 12 body fluids. Clin. Chem. 56: 1733-1741. 
Xiao C, Calado DP, Galler G, Thai TH, et al. (2007). MiR-150 controls B cell differentiation by targeting the transcription factor c-Myb. Cell 131: 146-159.

Zhou B, Wang S, Mayr C, Bartel DP, et al. (2007). miR-150, a microRNA expressed in mature B and T cells, blocks early B cell development when expressed prematurely. Proc. Natl. Acad. Sci. U. S. A. 104: 7080-7085.

Zhou Q, Li M, Wang X, Li Q, et al. (2012). Immune-related microRNAs are abundant in breast milk exosomes. Int. J. Biol. Sci. 8: 118-123. 